\title{
Skeletal muscle mitochondria as a target to prevent or treat type 2 diabetes mellitus
}

Citation for published version (APA):

Hesselink, M. K. C., Schrauwen-Hinderling, V., \& Schrauwen, P. (2016). Skeletal muscle mitochondria as a target to prevent or treat type 2 diabetes mellitus. Nature Reviews Endocrinology, 12(11), 633-645. https://doi.org/10.1038/nrendo.2016.104

Document status and date:

Published: 01/11/2016

DOI:

10.1038/nrendo.2016.104

Document Version:

Publisher's PDF, also known as Version of record

Document license:

Taverne

Please check the document version of this publication:

- A submitted manuscript is the version of the article upon submission and before peer-review. There can be important differences between the submitted version and the official published version of record.

People interested in the research are advised to contact the author for the final version of the publication, or visit the DOI to the publisher's website.

- The final author version and the galley proof are versions of the publication after peer review.

- The final published version features the final layout of the paper including the volume, issue and page numbers.

Link to publication

\footnotetext{
General rights rights.

- You may freely distribute the URL identifying the publication in the public portal. please follow below link for the End User Agreement:

www.umlib.nl/taverne-license

Take down policy

If you believe that this document breaches copyright please contact us at:

repository@maastrichtuniversity.nl

providing details and we will investigate your claim.
}

Copyright and moral rights for the publications made accessible in the public portal are retained by the authors and/or other copyright owners and it is a condition of accessing publications that users recognise and abide by the legal requirements associated with these

- Users may download and print one copy of any publication from the public portal for the purpose of private study or research.

- You may not further distribute the material or use it for any profit-making activity or commercial gain

If the publication is distributed under the terms of Article $25 \mathrm{fa}$ of the Dutch Copyright Act, indicated by the "Taverne" license above, 


\title{
REVIEWS
}

\section{Skeletal muscle mitochondria as a target to prevent or treat type 2 diabetes mellitus}

\author{
Matthijs K. C. Hesselink ${ }^{1,2}$, Vera Schrauwen-Hinderling ${ }^{1-3}$ and Patrick Schrauwen ${ }^{1,2}$
}

Abstract | Low levels of physical activity and the presence of obesity are associated with mitochondrial dysfunction. In addition, mitochondrial dysfunction has been associated with the development of insulin resistance and type 2 diabetes mellitus (T2DM). Although the evidence for a causal relationship between mitochondrial function and insulin resistance is still weak, emerging evidence indicates that boosting mitochondrial function might be beneficial to patient health. Exercise training is probably the most recognized promoter of mitochondrial function and insulin sensitivity and hence is still regarded as the best strategy to prevent and treat T2DM. Animal data, however, have revealed several new insights into the regulation of mitochondrial metabolism, and novel targets for interventions to boost mitochondrial function have emerged. Importantly, many of these targets seem to be regulated by factors such as nutrition, ambient temperature and circadian rhythms, which provides a basis for nonpharmacological strategies to prevent or treat T2DM in humans. Here, we will review the current evidence that mitochondrial function can be targeted therapeutically to improve insulin sensitivity and to prevent T2DM, focusing mainly on human intervention studies.

'Department of Human Biology and Human Movement Sciences, Maastricht University Medical Center,

Universiteitsingel 50, 6229

ER, Maastricht, Netherlands. ${ }^{2}$ NUTRIM, School for Nutrition and Translational Research in Metabolism, Maastricht University Medical Center, Universiteitsingel 50, 6229 ER, Maastricht, Netherlands. ${ }^{3}$ Department of Radiology, Maastricht University Medical Center, P. Debyelaan 25, 6229HX, Maastricht Netherlands.

Correspondence to P.S p.schrauwen@ maastrichtuniversity.nl

doi:10.1038/nrendo.2016.104 Published online 22 Jul 2016
Mitochondria are organelles that are essential for aerobic generation of ATP: the molecular currency that provides the chemical energy to fuel vital cellular processes. Mitochondrial number and function decline with normal ageing and compromised mitochondrial function is also associated with metabolic disorders such as cardiovascular disease and type 2 diabetes mellitus (T2DM), as well as various types of cancer ${ }^{1-3}$. Physical activity is probably the best-known strategy to improve mitochondrial function in metabolically active tissues. Many of the health benefits associated with physical activity might originate from exercise-induced improvements in mitochondrial function $^{4,5}$. The aim of this Review is to provide an overview of human studies targeting mitochondria with the objective to improve insulin sensitivity and prevent T2DM.

\section{Insulin resistance}

Insulin resistance refers to the blunted response of organs to insulin and is an important hallmark of the pathogenesis of T2DM. As a compensatory response to insulin resistance, plasma insulin concentrations rise in the early stages of T2DM. Insulin-mediated myocellular glucose uptake is key to maintaining normal glucose homeostasis, as approximately $80 \%$ of postprandial glucose clearance occurs within skeletal muscle ${ }^{6}$. Importantly, circulatory glucose levels are also affected by the release of glucose from the liver. Hepatic glucose output originates from hydrolysis of glycogen (the polysaccharide storage form of glucose) in the liver as well as from gluconeogenesis (de novo production of glucose from nonglucose-derived carbon precursors). Thus, to lower blood glucose levels, insulin promotes hepatic uptake of glucose while simultaneously inhibiting hepatic glucose production. In addition, insulin profoundly inhibits adipose tissue lipolysis, thereby lowering circulatory levels of fatty acids derived from adipose tissue in the postprandial state, which promotes the oxidation of glucose over other substrates. For acute regulation of glucose homeostasis, the stimulatory effect of insulin on glucose disposal and its inhibitory effect on hepatic glucose output are most important. However, long-term blunted inhibition of adipose tissue lipolysis in the insulin resistant state could result in storage of fat in nonadipose tissue and deleteriously affect cell function; this process is often referred to as lipotoxicity ${ }^{7}$.

\section{Insulin resistance and mitochondria}

Landmark studies relating insulin resistance to mitochondrial dysfunction reported reduced $\mathrm{NADH}_{2}-\mathrm{O}_{2}$ oxidoreductase activity (attributed to complex I) and structural mitochondrial aberrations ${ }^{8}$, with deficiency 


\section{Key points}

- Type 2 diabetes mellitus (T2DM) is associated with reduced mitochondrial function in the skeletal muscle and metabolic inflexibility

- Healthy mitochondria require a tight balance between mitochondrial biogenesis and mitophagy, as well as between mitochondrial ATP and reactive oxygen species production

- Exercise training is the intervention that most comprehensively improves the diverse aspects of mitochondrial function, along with whole-body and skeletal muscle insulin sensitivity

- Calorie restriction and calorie restriction mimetics are strong nonexercise tools to boost mitochondrial function, probably via the activation of an AMPK-NAD+-SIRT pathway

- Novel environmental and lifestyle factors, such as sleep and ambient temperature, might also affect mitochondrial function and insulin sensitivity in humans of subsarcolemmal mitochondrial function ${ }^{9}$, in patients with T2DM compared with healthy controls. Using microarray analyses, two independent studies almost simultaneously revealed a coordinated downregulation of genes that encode proteins involved in oxidative metabolism in muscle biopsy samples from patients with T2DM and from individuals with a positive family history of $\mathrm{T} 2 \mathrm{DM}^{10,11}$. These downregulated genes are under the control of a mitochondrial transcriptional cofactor known as peroxisome proliferator-activated receptor $\gamma$ co-activator $1 \alpha(\mathrm{PGC}-1 \alpha)$. A series of noninvasive ${ }^{31} \mathrm{P}$-magnetic resonance spectroscopy studies in elderly (61-84 years) patients ${ }^{12}$ and in young ( $26 \pm 7$ years; mean $\pm S D$ ) insulin-resistant offspring of patients with $\mathrm{T}_{2} \mathrm{DM}^{13}$ revealed a functional decline in in vivo ATPsynthase flux, determined by saturation transfer, which was identified as evidence for mitochondrial dysfunction. However, as mitochondrial activity is driven by cellular energy demand, the interpretation that these resting state observations truly reflect a mitochondrial defect that might contribute to insulin resistance is still subject to debate. In addition, from a methodological perspective, the interpretation of data from saturation transfer as a reflection of the rate of mitochondrial ATP synthesis is under discussion, owing to the overestimation of the ATP production using this method ${ }^{14}$. Furthermore, measurements in the resting state are expected to reflect resting metabolic rate rather than maximal capacity of mitochondrial function. Noninvasive measurement of the phosphocreatine resynthesis rate after exercise is used to investigate metabolism under stimulated conditions and is more widely accepted as a parameter of mitochondrial function ${ }^{14}$. These measurements have revealed that in patients with overt T2DM ${ }^{15}$, as well as in first-degree relatives of patients with $\mathrm{T} 2 \mathrm{DM}^{16}$, muscle oxidative capacity is compromised and can precede the development of frank T2DM. However, it should be noted that experimental animal models designed to directly examine the effect of mitochondrial dysfunction on insulin resistance (for example, dietary lipid overload ${ }^{17,18}$ or genetically compromised mitochondrial function ${ }^{19}$ ), do not necessarily show impeded insulin sensitivity. In humans, the debate regarding whether mitochondrial dysfunction is causal to insulin resistance or is the consequence of sustained overnutrition is still ongoing ${ }^{20,21}$. Notwithstanding this debate, multiple lines of evidence indicate that targeting mitochondrial biogenesis, structural integrity, function and/or number to maintain or improve insulin sensitivity, is worth exploring ${ }^{22,23}$. Of note, biguanides such as metformin, which are frequently used as a first-line treatment in T2DM, also seem to affect mitochondrial function ${ }^{24-27}$.

Mitochondrial dysfunction has not been clearly defined; in this Review, we refer to all aspects of mitochondrial dysfunction, including intrinsic mitochondrial dysfunction (characterized by the maintenance of mitochondrial mass but a decline in function of isolated mitochondria), reduced oxidative capacity and reduced mitochondrial mass.

Metabolic flexibility. How mitochondrial dysfunction could be causally linked to compromised insulin sensitivity remains to be determined. Although the most established feature of muscle mitochondria is the oxidative generation of ATP to fuel contractile activity of muscle fibres and intracellular signal transduction, details on quality control of mitochondria and mitochondrial maintenance of cellular and organelle function through processes such as autophagy or mitophagy were only described in the past few years ${ }^{28,29}$. Aberrations in autophagy and mitophagy have also been implicated in the aetiology of T2DM ${ }^{30}$. To adapt to oscillations in energy demand and substrate availability, healthy mitochondria can switch readily between oxidative metabolism of fatty acids and glucose. Although glucose can also be used to generate ATP nonoxidatively, catabolism of fatty acids requires proper mitochondrial function; therefore, if functional mitochondria are few and supply of glucose is sufficient (such as in the postprandial state), fatty acids compete with glucose for oxidative degradation ${ }^{31}$. With a reduced number of functional mitochondria, the flexibility to adjust substrate oxidation to substrate supply is diminished, a phenomenon referred to as metabolic inflexibility ${ }^{32}$. Indeed, the inflexibility to adapt substrate selection from predominantly fatty acids in the fasted state to glucose oxidation in the postprandial, high-insulin state is a common characteristic of insulin-resistant muscle ${ }^{33}$ and is often observed in conjunction with mitochondrial dysfunction in patients with T2DM.

At the cellular level, metabolic flexibility can be explained by the competition of acetyl coenzyme A (acetyl-CoA) derived from glycolysis or from $\beta$-oxidation for entrance into the tricarboxylic acid cycle; accumulation of acetyl-CoA, for example derived from high $\beta$-oxidation fluxes, can inhibit pyruvate dehydrogenase activity and reduce glycolytic flux (FIG. 1). In an attempt to maintain low mitochondrial acetyl-CoA levels and to sustain glycolytic flux, acetyl-CoA can be converted to acetylcarnitine by the mitochondrial matrix enzyme carnitine acetyltransferase (CrAT). However, in a state of insulin resistance, the availability of free carnitine is decreased ${ }^{34}$ and the activity of CrAT is hampered ${ }^{35}$. In patients with obesity or T2DM, acetylcarnitine levels are low and CrAT activity correlates strongly and positively with insulin sensitivity $^{36}$. This mechanism might underlie compromised postprandial glucose oxidation in the insulin resistant 


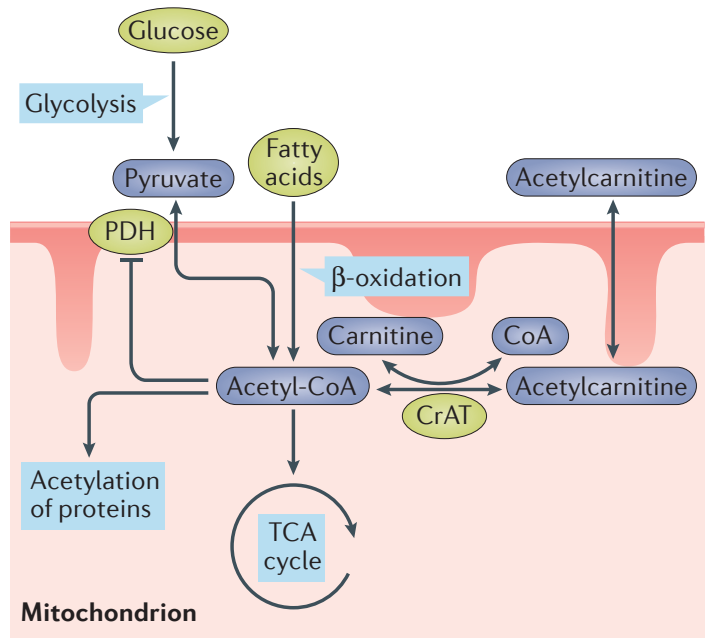

Figure 1 | The conversion of acetyl-CoA to acetylcarnitine by carnitine-acyltransferase. Excess acetyl units can be exported from the mitochondria to prevent mitochondrial acetyl-coenzyme A (acetyl-CoA) accumulation. Accumulation of acetyl-CoA inhibits glucose metabolism by inhibiting pyruvate dehydrogenase $(\mathrm{PDH})$, the enzyme that acts as the gate keeper for entry of glycolysis-derived pyruvate into the mitochondria and hence a key regulator for oxidative glucose degradation. By transferring acetyl units to acetyl-carnitine, carnitine-acyltransferase (CrAT) alleviates the inhibitory effect of excess mitochondrial acetyl-CoA on the flux through the $\mathrm{PDH}$ complex, facilitating oxidative glucose catabolism. TCA, tricarboxylic acid.

state and aids understanding of the observed metabolic inflexibility, as reduced acetylcarnitine production would lead to the accumulation of acetyl-CoA and consequently inhibit conversion of pyruvate, impeding the use of glucose as a respiratory substrate. Conversely, an abundance of mitochondria with high CrAT activity promotes acetylcarnitine generation, which enables buffering of fluctuations in substrate availability and thereby improves metabolic flexibility and glucose tolerance.

Mitochondrial ROS production. Superoxide and other reactive oxygen species (ROS) are generated as inevitable by-products of mitochondrial respiration. Excess ROS production, which could be due to dysfunctional mitochondria, in the absence of sufficient antioxidant capacity might result in lipid peroxidation and other forms of oxidative stress such as damage to nuclear and mitochondrial $\mathrm{DNA}^{37}$, which in turn might also directly contribute to the development of insulin resistance ${ }^{38}$.

A high supply of electron donors to the electron transport chain without a concomitant increase in ATP synthase flux results in a high proton gradient across the inner mitochondrial membrane and augments ROS production. By adjusting the efficiency of ATP generation within the mitochondrial machinery, mitochondria can regulate cellular energy turnover and superoxide production. The underlying process responsible for reducing the efficiency of ATP production (and hence modulating energy turnover, superoxide production and heat production) is referred to as mitochondrial uncoupling. Mitochondrial uncoupling can occur in a tightly regulated way, for example, uncoupling occurs upon cold exposure in brown adipose tissue (BAT) via the activation of uncoupling protein 1 (UCP1; BOX 1), a transmembrane protein that decreases the proton gradient generated through oxidative phosphorylation. In other tissues, mitochondria can also be uncoupled by fatty acids ${ }^{39}$, although the exact mechanism is not known. However, it remains to be elucidated to what extent uncoupling of mitochondria in other cell types contributes to regulation of ROS production and/or energy turnover. Clearly, mitochondrial efficiency and mild uncoupling are attractive therapeutic targets to increase energy turnover and alleviate ROS production, thereby promoting glucose catabolism and thus insulin sensitivity (BOX 1). However, it should be noted that uncontrolled uncoupling is an unwanted process as it could result in a life-threatening increase in body temperature and critically low ATP levels ${ }^{40}$.

Mitochondrial health, maintenance and dynamics. According to the currently widely supported endosymbiotic theory, mitochondria in eukaryotic cells originate from symbiosis of single-celled organisms and proteobacteria $^{41}$. Hence, mitochondria carry their own circular $\mathrm{DNA}^{42}$ that encodes 22 transfer RNAs and only 13 proteins, some of which are required to maintain respiratory activity. A short open reading frame in the mitochondrial DNA (mtDNA) has been identified that encodes at least two mitochondria-derived signalling peptides that have been implicated in insulin resistance: humanin ${ }^{43}$ and mitochondrial-derived peptide MOTS- $\mathrm{c}^{44}$. The majority of the mitochondrial proteins are nuclear-encoded and rely on post-translational import into the mitochondrion ${ }^{45}$. For nuclear-encoded genes, induction of gene expression, protein synthesis, processing and mitochondrial import relies on cellular translational machinery, but must occur in an orchestrated manner with replication of mtDNA and subsequent mitochondrial protein synthesis. This need for orchestration is exemplified by the observation that transcription of mtDNA genes requires the nuclear-encoded mitochondrial transcription factor $\mathrm{A}^{46}$.

The master regulator of this complex process is PGC-1a, which interacts with a variety of nuclear transcription factors involved in maintenance of the oxidative phenotype, such as nuclear hormone receptors, nuclear respiratory factors and muscle-specific transcription factors. Mice overexpressing PGC-1 $a$ have more muscle fibres that are dependent on oxidative metabolism and a massive increase in mitochondrial mass compared with wild-type mice ${ }^{47}$. Mitochondria isolated from PGC-1 $\alpha$ overexpressing mice have an increased capacity to oxidize lipids compared with wild-type mice ${ }^{48}$. Lifelong overexpression of PGC-1a in mouse skeletal muscle reduces insulin sensitivity ${ }^{49}$ (unless energy demand is increased ${ }^{50}$ ); conversely, transfection of human muscle cells with PGC-1 $1 \alpha$ yields an insulin-sensitive phenotype similar to that which is observed in highly trained athletes, with increased mitochondrial number and sequestration of excess lipid in inert cellular lipid droplets ${ }^{51}$. Additionally, a number of oxidative genes under control 


\section{Box 1 | Mitochondrial uncoupling}

Mitochondrial uncoupling is the process by which the proton motive force built up by the electron transport chain is uncoupled from oxidative phosphorylation by proton leak or by uncoupling proteins in the inner mitochondrial membrane. In brown adipose tissue, this process is tightly regulated and facilitated by uncoupling protein 1 , which is specific for brown and beige adipocytes. Regulated mitochondrial uncoupling by uncoupling proteins dissipates the proton gradient to generate heat rather than ATP and is, therefore, suggested to be involved in the regulation of body temperature. In other tissues, uncoupling proteins are also present but might have distinct functions. Thus, in the case of excess substrate availability, the supply of NADH or FADH ${ }_{2}$ to the electron transport chain is increased and hence the proton gradient is heightened. If cells are inactive and ADP levels and ATP turnover are low, then proton motive force is not dissipated by the ATP synthase but by proton leak only; thus, electron flow through the electron transport chain is substantially reduced, whereas the proton motive force is high. Under these conditions, the electrons donated to $\mathrm{O}_{2}$ result in generation of superoxide and $\mathrm{H}_{2} \mathrm{O}_{2}$, which results in a condition of oxidative stress. Modest mitochondrial leak or mild mitochondrial uncoupling via uncoupling proteins such as uncoupling protein 3 can dissipate the proton motive force and hence serve to alleviate oxidative stress ${ }^{159}$. In most cell types, coupling of mitochondria is not $100 \%$ efficient but ranges between $70 \%$ and $90 \%$ and the relationship between proton motive force and superoxide production is fairly steep. Thus, mild uncoupling can be a tool to alleviate oxidative stress at the expense of bioenergetic efficiency ${ }^{160}$.

of PGC-1a undergo concerted downregulation in patients with increased susceptibility to $\mathrm{T} 2 \mathrm{DM}^{10,11}$. Furthermore, insulin sensitizing treatments such as exercise training ${ }^{52}$ and rosiglitazone ${ }^{53}$ result in the parallel restoration of both PGC-1 $\alpha$ and insulin sensitivity, which suggests that PGC- $1 \alpha$ is an important therapeutic target for the improvement of mitochondrial function and insulin sensitivity. Interestingly, phosphorylation and deacetylation of PGC-1 $\alpha$ both regulate its activity. Although phosphorylation of PGC-1a seems to be mediated by AMP activated protein kinase (AMPK), which is activated by a drop in the ATP:AMP ratio, deacetylation depends on $\mathrm{NAD}^{+}$-mediated activation of NAD-dependent protein deacetylase sirtuin-1 (SIRT1) $)^{54}$, both of which are factors that have been associated with improved insulin sensitivity.

Downstream of PGC-1 $1 \alpha$ and other members of the PGC1 transcription family, activation of a protein involved in regulating mitochondrial turnover, mitofusin-2, maintains the mitochondrial network ${ }^{55}$, which is a process that is also sensitive to metabolic perturbations. Although mitochondria are classically portrayed as solitary organelles, mitochondria present as a network of interconnected organelles in very large, metabolically dynamic cells such as those of the skeletal muscle. The network facilitates transmission of oxygen from the capillaries to the core of the myocyte (FIC. 2a) and creates electrical connectivity for rapid conductance of mitochondrial membrane potential ${ }^{56}$ throughout the lengthy muscle cells (FIG. 2b). Maintenance of the mitochondrial network requires a constant antagonist balance between mitochondrial fusion and fission ${ }^{57}$, collectively referred to as mitochondrial dynamics. These dynamics are essential for quality control of mitochondria. Using mitochondrial fusion, damaged mtDNA is exchanged with intact mtDNA from neighbouring mitochondria. Conversely, mitochondrial fission enables the removal of impaired or redundant mitochondria by an autophagic process referred to as mitophagy, thus preventing proliferation of mutated $\mathrm{mtDNA}^{57}$.

Dynamics of mitochondria could also have a role in the acute regulation of the efficiency of mitochondrial ATP generation. Mitochondrial fusion encompasses fusion of the outer mitochondrial membrane, regulated by mitofusin- 1 and mitofusin-2, and fusion of the inner mitochondrial membrane, regulated by dynamin-like $120 \mathrm{kDa}$ protein (also known as optic atrophy protein 1 ; OPA1 $)^{58}$. Mitochondrial fusion achieved by induction of mitofusin-2 is transiently triggered upon either absolute nutrient deprivation, indicated by a cellular drop in ATP levels, or by relative nutrient deprivation, characterized by increased demand for ATP generation ${ }^{59,60}$. Under these conditions, OPA1 promotes tightening of mitochondrial cristae width and assembly of ATP synthase, which in turn promotes efficiency of ATP production ${ }^{61}$. Conversely, excess supply of mitochondrial substrate increases proton motive force (BOX 2) and promotes mitochondrial production of ROS. These circumstances trigger mitochondrial fission ${ }^{62}$ by binding of dynamin1-like protein (DNML1) to its outer membrane receptor mitochondrial fission 1 protein (FIS1) ${ }^{63}$.

The process of mitochondrial fusion has also been proposed to lead to mitochondrial uncoupling and thereby to dissipation of energy, at least in mouse, rat and cell models. In addition, experiments in BAT mitochondria have shown that excess nutrient supply induces mitochondrial fragmentation, with a concomitant drop in mitochondrial efficiency and increased fatty-acid-mediated uncoupling ${ }^{64}$. Thus, continuous oversupply of nutrients might induce fission, which - in the absence of opposed fusion - translates into mitochondrial uncoupling and energy dissipation with increased energy expenditure as a consequence. Interestingly, a low metabolic rate in humans, such as that detected during sleep, has been associated with the development of obesity and metabolic diseases; skeletal muscle is considered to be a major determinant of human resting metabolic rate ${ }^{65}$. So far, the processes underlying the variability in human energy metabolism have not been revealed, although the discovery of UCP3, a homologue of UCP1, in human skeletal muscle resulted in speculations that these inner mitochondrial membrane proteins might be responsible for the variation in metabolic rate ${ }^{66}$. However, no evidence is currently available to suggest that these uncoupling proteins can be considered direct determinants of human metabolic rate.

To speculate that the dynamic process of mitochondrial fusion and fission might underlie some of the variance in human metabolic rate is tempting. Under conditions of transiently increased energy demand, fusion generates a mitochondrial network that is well equipped for efficient ATP generation while simultaneously providing means to maintain mtDNA quality. Under conditions of a transient energy oversupply, mitochondrial fission renders mitochondria inefficient and excess substrates can be consumed as a counterbalance response, while simultaneously relieving ROS production. Disturbances in this process of mitochondrial dynamics might, therefore, 
a

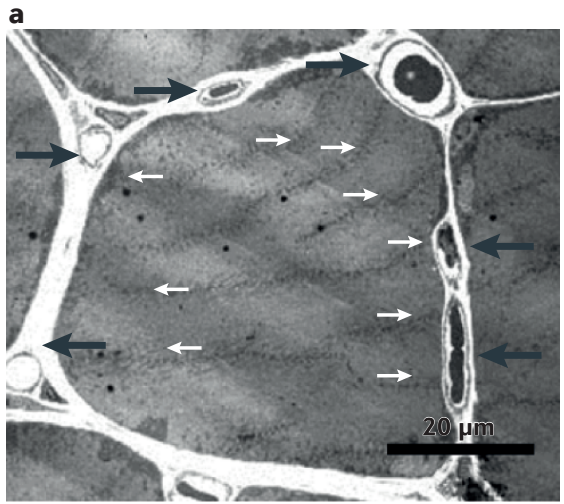

b

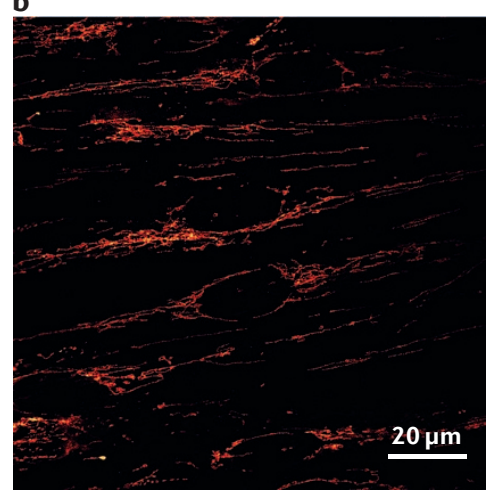

Figure 2 | Mitochondrial network morphology. a | Cross-sectional transmission electron micrograph of a skeletal muscle cell in a rat soleus muscle showing penetration of a mitochondrial network (black arrows) from the site of oxygen and substrate supply (the capillaries, grey arrows) towards the core of the muscle cell. Scale bar represents $20 \mu \mathrm{m} . \mathbf{b}$ | Confocal microscopy image of the extension of the mitochondrial network along cultured human myotubes, taken in the longitudinal plane. The mitochondrial network was visualized by staining against mitochondrial import receptor subunit TOM20 homologue. The signal is pseudocoloured in red, areas with high mitochondrial density display orange and yellow. Scale bar represents $20 \mu \mathrm{m}$.

limit the regulation of mitochondrial ATP efficiency and dissipation of excess energy as well as disrupting the quality control of mitochondria. As a result of these changes, mitophagy will be promoted, and mitochondrial content and function might become compromised ${ }^{67}$. Indeed, sustained defects in mitochondrial dynamics can compromise mitochondrial quality and are associated with insulin resistance; furthermore, studies in mice and in rat muscle cells have revealed that repression of mitofusin-2 expression reduced glucose oxidation and impaired glucose homeostasis ${ }^{68}$.

Conversely, mitofusin-2 gain-of-function experiments in cell models showed an abundance of perinuclear mitochondria and enhanced glucose oxidation ${ }^{69}$. A defect in mitofusin-2 is, however, not causally linked to insulin resistance, as is exemplified by the observation that humans carrying a defect in the gene that encodes mitofusin-2 (resulting in a neurodegenerative disorder known as Charcot-Marie-Tooth disease) are not characterized by aberrant glucose handling or insulin resistance ${ }^{70}$. Expression of mitofusin-2 in skeletal muscle is lowered in patients with obesity and T2DM; moreover, mitofusin-2 expression levels correlated positively with insulin sensitivity in patients with T2DM. This finding indicates that induction of mitofusin-2 could promote mitochondrial fusion in order to surmount increases in fission mediated by the oversupply of nutrients, maintaining or improving insulin sensitivity as a consequence ${ }^{71}$. Induction of mitochondrial fission by nutrient oversupply promotes mitochondrial dysfunction and has been causally linked to insulin resistance in $\mathrm{C} 2 \mathrm{C} 12$ skeletal muscle cells as well as in $\mathrm{H} 9 \mathrm{c} 2$ cardiac myocytes ${ }^{72,73}$. So far, non-lethal mutations in genes involved in mitochondrial fission have not been identified ${ }^{74}$; hence, data in humans on the role of mitochondrial fission and insulin resistance is lacking, but sustained disturbances in the delicate balance of fission and fusion could feasibly impede mitochondrial quality control and respiratory capacity.

Although these data do not presently reveal a unifying mechanism that links mitochondrial function to insulin sensitivity, it seems safe to state that by targeting metabolic flexibility, ROS stress and mitochondrial quality control (through the balancing of mitochondrial biogenesis, autophagy, mitophagy, fission and fusion), aspects of mitochondrial function can be improved, all of which are linked to insulin sensitivity.

\section{Effects of exercise}

Exercise training is one of the most potent ways to promote increased muscle oxidative capacity, and is considered one of the best strategies to prevent and treat T2DM. A pioneering study in the early 1980s showed that a training programme of 16 weeks improved peripheral insulin sensitivity in human muscle of insulin resistant patients with type 1 diabetes mellitus in parallel with increased mitochondrial oxidative enzyme capacity ${ }^{75}$, preceding the discovery of the exercise responsive solute carrier family 2, facilitated glucose transporter member 4 (GLUT-4) in 1987 (REF. 76). A plethora of papers have examined the effect of a wide range of exercise training programmes on markers of mitochondrial function and insulin sensitivity in humans $s^{52,77-86}$. Although these studies consistently report exercise-mediated improvements in mitochondrial function and insulin sensitivity (TABLE 1), it is also clear that a mutual improvement in both of these factors is not necessarily guaranteed.

One potential confounder contributing to the dissociation between improvements in mitochondrial function and improved insulin sensitivity is the duration of T2DM at the time of intervention. Indeed, it was observed that baseline maximal oxygen consumption correlates inversely with the duration of $\mathrm{T}_{2} \mathrm{DM}^{87}$. In addition, an increase in mitochondrial function upon treatment with the insulin-sensitizing drug rosiglitazone, which was measured by the increase in expression of proteins in the electron transport chain and increased citrate synthase activity, was only observed in patients with the best preserved basal aerobic capacity ${ }^{87}$. However, whether this outcome also applies to exerciseinduced effects on mitochondrial function is presently unknown. In a short-term high intensity training programme, mitochondrial function improved similarly in individuals without T2DM and in the offspring of mothers with T2DM, whereas insulin sensitivity improved only in people without diabetes mellitus ${ }^{84}$. A more classic endurance type of training programme, consisting of three 20-35 min sessions of cycling exercise per week for 10 weeks, in patients with T2DM and BMI-matched normoglycaemic controls also improved mitochondrial function, but these changes were not quantitatively correlated with improvements in insulin sensitivity ${ }^{82}$.

Another reason for this apparent lack of correlation between changes in mitochondrial function and insulin sensitivity might originate from the definition of insulin sensitivity, which consists of oxidative (stimulation of glucose oxidation by insulin) and nonoxidative (stimulation of glycogen storage by insulin) insulin-stimulated glucose uptake. Thus, in a training study in patients with 


\section{Box 2 | Mitochondrial energy production}

The primary task of skeletal muscle mitochondria is to generate ATP. To do so, mitochondria need reducing equivalents (such as $\mathrm{NADH}$ and $\mathrm{FADH}_{2}$ ) and $\mathrm{O}_{2}$. Reducing equivalents predominantly originate from the catabolism of glucose and fatty acids by glycolysis, $\beta$-oxidation and the tricarboxylic acid cycle. Mitochondrial ATP generation requires potential energy for the reaction of inorganic phosphate with ADP to generate ATP and $\mathrm{H}_{2} \mathrm{O}$. The potential energy is derived from building a proton gradient across the inner mitochondrial membrane by the electron transport chain. In the electron transport chain, $\mathrm{NADH}$ is reduced by complex I (also known as NADH dehydrogenase). Complex I facilitates pumping of protons across the inner mitochondrial membrane and transfers electrons to coenzyme Q. Complex II (also known as succinate dehydrogenase) and the ubiquinone-coenzyme $\mathrm{Q}$ cycle transfers electrons from $\mathrm{FADH}_{2}$, which can originate from tricarboxylic acid cycling, as well as from anaplerotic reactions (chemical reactions that form intermediates of a metabolic pathway) of amino-acid-derived carbons such as succinyl-coenzyme A into succinate dehydrogenase. Complex II has no proton pumping capacity. Complex III has proton pumping capacity and contains the ultimate electron acceptor cytochrome $\mathrm{C}$. Complex IV (also known as cytochrome $\mathrm{C}$ oxidase) also pumps protons across the inner mitochondrial membrane upon acceptance of the electrons offered by cytochrome $\mathrm{C}$. The activity of complex IV is dependent on $\mathrm{O}_{2}$, as $\mathrm{O}_{2}$ is the final electron acceptor. In the presence of hydrogen and upon acceptance of two electrons, $\mathrm{O}_{2}$ is reduced to $\mathrm{H}_{2} \mathrm{O}$ at complex IV. As the permeability of the intact inner mitochondrial membrane for protons is minimal, proton pumping results in a proton gradient and hence increases proton motive force across the membrane. In skeletal muscle mitochondria, a protein complex that spans the inner mitochondrial membrane, complex V (also known as ATP synthase), utilizes energy from the proton gradient to catalyse ATP production and drive the high ATP:ADP ratio in cells. This process of oxidative phosphorylation is driven by high ATP turnover.

T2DM and normoglycaemic controls matched for BMI, age and physical fitness, our group observed that training increased protein expression of electron transport chain complex I-V, and ADP-driven state 3 respiration (mitochondrial oxygen consumption upon the addition of substrates and ADP) on a variety of substrates, such as glutamate and succinate, and also improved phosphocreatine recovery rate after exercise in both groups. In patients with T2DM, we detected restoration of mitochondrial function to levels observed in normoglycaemic control individuals $s^{52,86}$. Interestingly, dissecting oxidative from nonoxidative glucose disposal revealed that oxidative glucose disposal was restored to control values, in line with restoration of mitochondrial function, whereas nonoxidative glucose disposal was not restored ${ }^{52}$. This observation indicates that an exercise-induced improvement in mitochondrial function improves the oxidative part of insulin sensitivity, but not necessarily the nonoxidative part, and thereby aids our understanding of the reported dissociation between training-mediated improvements in mitochondrial function and insulin sensitivity observed in some studies. Notably, metabolic flexibility, which has also been hypothesized as a putative mechanism connecting mitochondrial dysfunction and insulin resistance, was similarly restored upon exercise training in this particular study ${ }^{52}$. Training-mediated improvements in metabolic flexibility and concomitant improvements in insulin sensitivity have also been observed in patients with impaired fasting glucose and impaired glucose tolerance ${ }^{88}$, which suggests that improving metabolic flexibility by physical activity can be beneficial in preventing the transition from an impaired glucose tolerant to an insulin resistant state.
Encouragingly, metabolic flexibility and markers of mitochondrial function were both shown to correlate with levels of habitual physical activity ${ }^{89,90}$. This finding endorses the feasibility of targeting metabolic flexibility by promoting habitual physical activity to improve insulin sensitivity.

Alternatively, exercise training could affect insulin sensitivity via alteration of mitochondrial ROS production. In healthy women, an 8-week exercise training programme improved insulin sensitivity, as defined by HOMA, and the balance of oxidative stress markers to antioxidant status markers also improved ${ }^{91}$. In addition, women with insulin resistance and polycystic ovary syndrome who underwent a 12-week endurance training programme had reduced mitochondrial $\mathrm{H}_{2} \mathrm{O}_{2}$ production, increased catalase activity and reduced oxidative DNA damage subsequent to the training programme, in parallel with improvements in insulin sensitivity ${ }^{92}$. An interesting mechanistic explanation for how exercise training might alleviate $\mathrm{H}_{2} \mathrm{O}_{2}$ production in diabetic muscle is provided by studies showing that the bioactive lipid derivative palmitoyl coenzyme A (palmitoyl-CoA) diminishes mitochondrial influx of ADP in human diabetic muscle ${ }^{93}$. As a result of this inhibition of ADP transport, dissipation of the proton motive force via ATP synthesis is reduced, with increased $\mathrm{H}_{2} \mathrm{O}_{2}$ production as a consequence. Interestingly, exercise training reduces the inhibitory effect of palmitoyl-CoA on mitochondrial ADP influx, thereby promoting ATP synthesis with concomitant reduction of proton motive force and alleviation of oxidative stress ${ }^{93}$. ADP/ATP translocase 1, a mitochondrial protein that is extensively acetylated in human diabetic muscle, facilitates mitochondrial ADP-ATP exchange and hence the capacity of ATP synthase to dissipate the proton gradient and alleviate oxidative stress ${ }^{94}$. Interestingly, the level of acetylation of mitochondrial proteins is negatively correlated with glucose disposal rates, whereas exercise-induced reduction of ADP/ATP translocase 1 acetylation correlates with improvements in glucose disposal ${ }^{94}$. Moreover, our group found that ADP/ATP translocase 1 protein expression and the sensitivity of ADP/ATP translocase 1 to fatty acids resulting in fatty-acid-induced uncoupling was heightened in trained athletes compared with lean, sedentary controls, and correlated with glucose disposal ${ }^{95}$. Interestingly, using a noninvasive NMR-based approach to calculate the ratio of ATP synthesis to tricarboxylic acid cycle flux in skeletal muscle as a measure of mitochondrial coupling efficiency in trained athletes showed elevated mitochondrial uncoupling in exercise-trained muscle ${ }^{96}$. Although direct evidence that exercise training alleviates ROS production and thereby promotes insulin sensitivity is limited, these studies suggest that the beneficial effects of exercise on insulin sensitivity can also be linked with exercisemediated improvements in respiratory substrate handling that alleviates ROS production via regulated mitochondrial energy dissipation (BOX 2).

Data from animal studies have revealed that targeting mitochondrial dynamics by exercise training could lead to profound mitochondrial remodelling ${ }^{97}$. However, only a few human studies have examined the effect of exercise 
Table 1 | Effect of exercise training on mitochondrial function and glucose homeostasis in humans

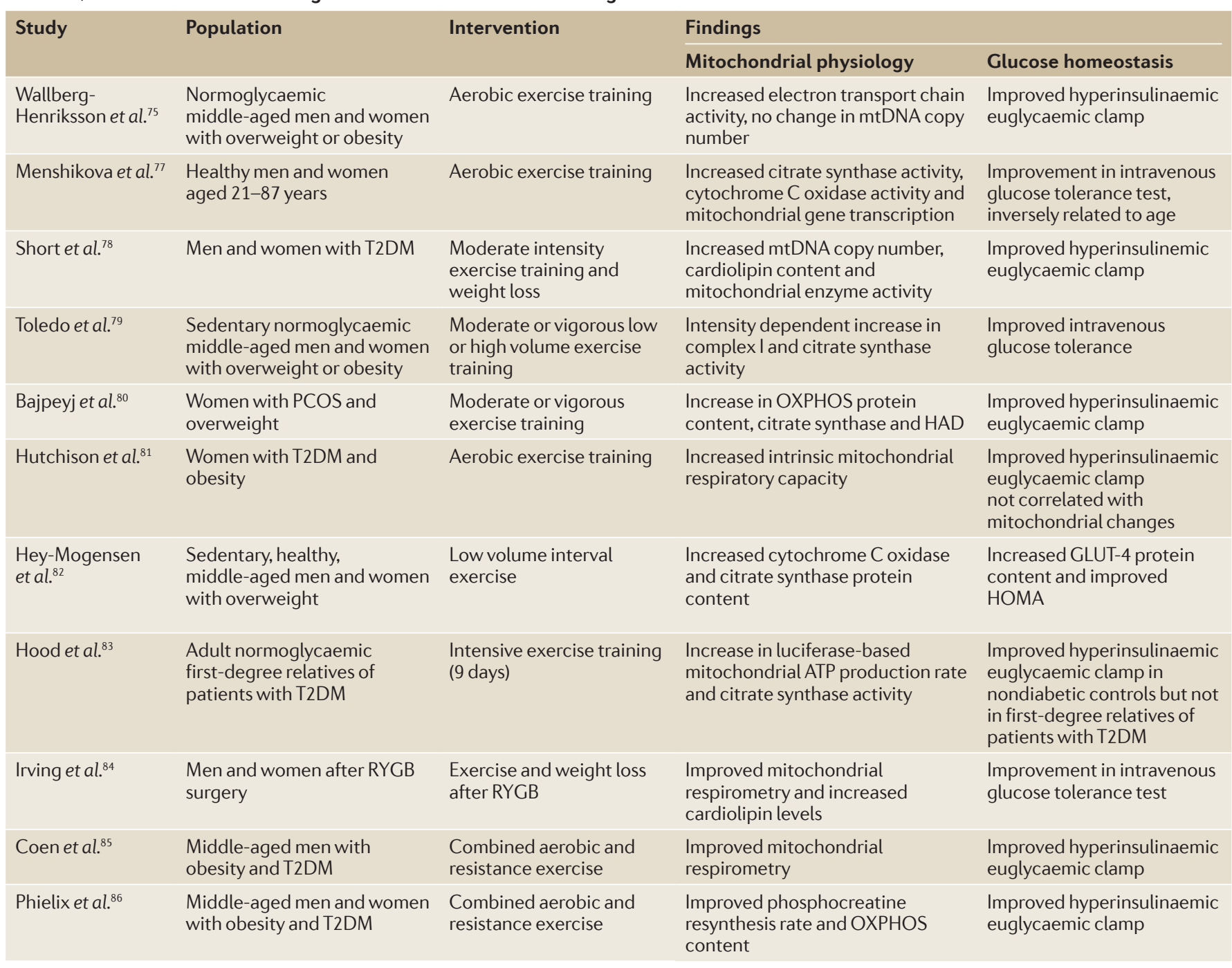

GLUT-4, solute carrier family 2, facilitated glucose transporter member 4; HAD, 3-hydroxy-acylCoA-dehydrogenase; mtDNA, mitochondrial DNA; OXPHOS, oxidative phosphorylation; PCOS, polycystic ovary syndrome; RYGB, Roux-en-Y gastric bypass; T2DM, type 2 diabetes mellitus.

training on mitochondrial dynamics and its putative relationship with insulin sensitivity. In trained cyclists, acute exercise induces increased expression of genes that encode proteins involved in mitochondrial dynamics, such as mitofusin-1 and mitofusin-2 (REF. 98). In healthy sedentary patients, as well as in patients with chronic heart failure, endurance training induced increased expression of genes involved in mitochondrial fusion (such as the gene that encodes mitofusin-2) and fission (such as the gene that encodes DNML1) ${ }^{99}$, which is suggestive of training-mediated activation of mitochondrial remodelling (although this process was not directly measured). In patients newly diagnosed with T2DM, baseline gene expression of markers of mitochondrial dynamics are reduced compared with control individuals with obesity. However, only individuals with obesity and not patients with T2DM responded to exercise training, with improvements in the mitochondrial remodelling machinery ${ }^{100}$. At present, the only study linking exercise-mediated changes in mitochondrial dynamics to markers of insulin sensitivity was performed in adults with insulin resistance and obesity, and showed that exercise training reduced phosphorylation of DNML1 (thereby inhibiting fission) whereas markers of mitochondrial fusion (such as mitofusin-1, mitofusin-2 and OPA1) showed a trend towards elevation. Moreover, the reduction in DNML1mediated fission activity correlated with the trainingmediated improvement in insulin sensitivity ${ }^{101}$. Thus, although conceptually attractive and supported by animal data, the notion that mitochondrial remodelling upon exercise training might contribute to improved mitochondrial function and insulin resistance remains to be firmly supported by experimental data in humans.

\section{Nonexercise mediated effects}

In addition to the well-known effects of exercise on mitochondrial function, studies in the past two decades have also investigated nonexercise means to promote many aspects of mitochondrial function. Although the number of suggestions for putative targets to modulate 
mitochondrial function in a nonexercise manner from animal studies is high, only a few of these targets have been confirmed or studied in humans.

Calorie restriction, $\mathrm{NAD}^{+}$metabolism and sirtuins. In a wide range of species, including humans, calorie restriction is effectively the sole intervention with proven effects of promoting increased lifespan. Although the underlying mechanisms for these calorie-restriction-mediated effects are not completely understood, many of the suggested processes involve mitochondria ${ }^{102}$. Investigations in humans, however, are limited. Weight loss has been recognized to improve insulin sensitivity, but studies examining the effects of weight loss often practice a rigid restriction in energy intake that is not comparable to the calorie restriction regimes performed in other species. One of the best controlled and longest calorie restriction trials in humans, featuring a $25 \%$ reduction of baseline energy requirements, reported reduced fasting insulin levels ${ }^{103}$ and increased gene expression of PGC-1 $\alpha$, mitochondrial transcription factor A and SIRT1, along with increased mtDNA content in skeletal muscle after calorie restriction $^{104}$, which indicates that mitochondrial remodelling might also be part of the adaptive process to calorie restriction in humans. These studies suggest a central role for SIRT1 and its activator $\mathrm{NAD}^{+}$as molecular regulators of the beneficial effects of calorie restriction and increased insulin sensitivity ${ }^{105}$.

Profound fluctuations in $\mathrm{NAD}^{+}$are associated with increased energy expenditure (BOX 1). The rate-limiting step for the biosynthesis of $\mathrm{NAD}^{+}$is a reaction catalysed by nicotinamide phosphoribosyltransferase (NAMPT). Indeed, NAMPT ${ }^{106,107}, \mathrm{NAD}^{+}$, precursors of $\mathrm{NAD}^{+}$ (REF. 108) and SIRT1 (REF. 109) have all successfully been targeted to promote deacetylase activity and insulin

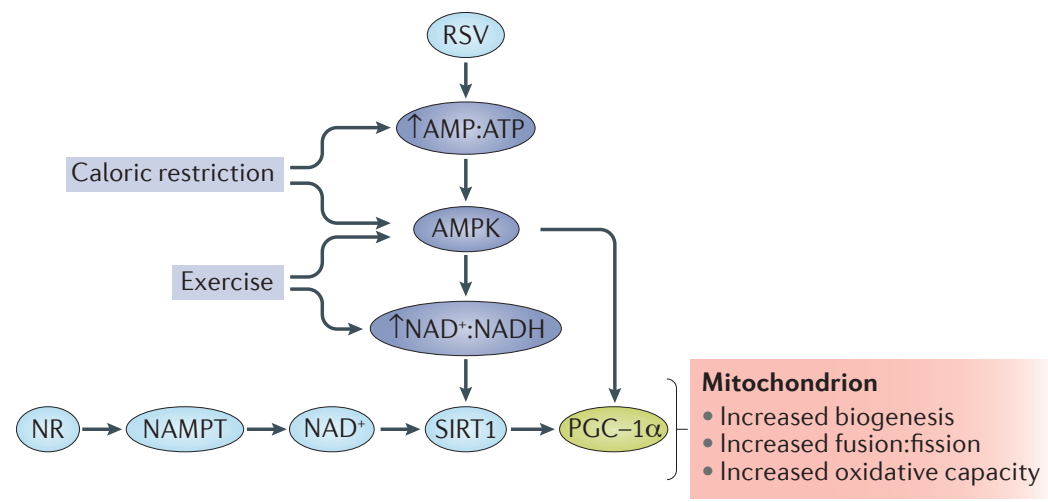

Figure $3 \mid$ Schematic overview of the role of $\mathrm{NAD}^{+}$in the regulation of mitochondrial metabolism. Exercise and caloric restriction directly and indirectly activate AMPK, thereby activating peroxisome proliferator-activated receptor $\gamma$ co-activator $1 \alpha$ (PGC-1a) while simultaneously promoting an increased $\mathrm{NAD}^{+}$to $\mathrm{NADH}$ ratio and activating sirtuin 1 (SIRT1). Nutritional compounds such as resveratrol (RSV) can also activate SIRT1 through this route, whereas $\mathrm{NAD}^{+}$precursors, such as nicotinamide riboside (NR), might directly elevate $\mathrm{NAD}^{+}$levels through catalysis by nicotinamide phosphoribosyltransferase (NAMPT) and promote SIRT1 activity. The downstream outcome of activating these pathways converges onto an improved mitochondrial phenotype by multiple routes. sensitivity in animals. Although the gene expression of SIRT1 is reduced in patients with T2DM compared with healthy individuals ${ }^{110}$, human intervention studies have so far been limited. In addition, a phase II study using a novel SIRT1 activator failed to show beneficial effects on fasting and postprandial glucose metabolism ${ }^{111}$. The polyphenolic compound resveratrol was identified as a small-molecule activator of SIRT1 (REF. 112), and has been widely studied in humans, with $>10$ human clinical resveratrol intervention trails performed to date, examining markers of insulin sensitivity as the primary outcome ${ }^{113}$. Although promising results have been obtained, the outcomes of these studies are not unequivocal, which might originate from differences in duration and dosing levels of resveratrol between the trials. Unfortunately, only a few human studies have investigated whether resveratrol administration results in activation of the AMPK-SIRT1 1 axis $^{114}$, as was suggested by findings from animal model studies. For further discussion on the effects of resveratrol on human metabolism we refer the reader a previous review ${ }^{115}$.

In a more direct way to affect mitochondrial metabolism, $\mathrm{NAD}^{+}$levels could be increased by supplementation of the natural $\mathrm{NAD}^{+}$precursor nicotinamide riboside. In mice, this approach resulted in improved oxidative capacity and alleviation of high-fat-diet-induced metabolic aberrations ${ }^{116}$, and both prevented and reverted development of nonalcoholic fatty liver disease in mice via induction of an imbalance in mitochondrial-encoded proteins compared with nuclear-encoded mitochondrial proteins in a SIRT1 and SIRT3-dependent manner ${ }^{117}$. The likelihood that nicotinamide riboside supplementation is also beneficial for humans was underscored by a 2015 paper that reported ex vivo nicotinamide riboside treatment of peripheral blood mononuclear cells from healthy volunteers, which revealed increased mitochondrial respiratory capacity, increased antioxidant capacity and reduced mitochondrial ROS production upon nicotinamide riboside treatment ${ }^{118}$. Furthermore, using an indirect approach, our group showed that boosting $\mathrm{NAD}^{+}$ levels via the $\mathrm{NAD}^{+}$precursor acipimox robustly induced an imbalance in mitochondrial-encoded and nuclearencoded mitochondrial proteins and improved muscle mitochondrial respiration in patients with $\mathrm{T} 2 \mathrm{DM}^{119}$ Clearly, more human intervention studies are needed to reveal the true potential of targeting the AMPK-NAD ${ }^{+}$ SIRT1 pathway (FIG. 3) to improve insulin sensitivity, but the data available so far are certainly promising.

Circadian rhythms and sleep. Evidence from epidemiological ${ }^{120,121}$ and intervention studies ${ }^{122,123}$ increasingly implicate a role for circadian rhythms and sleep in maintenance of glucose homeostasis. Night shift workers (who represent $\sim 10 \%$ of the working global population) have an increased risk of developing T2DM and cardiovascular disease ${ }^{124}$. Furthermore, laboratory-induced circadian misalignment, caused by $28 \mathrm{~h}$ days, results in disturbances in glucose homeostasis in healthy individuals ${ }^{123}$. Most importantly, circadian misalignment not only affects a small part of the general population who undertake night shifts, but also the larger part of the 
Cellular time keeping

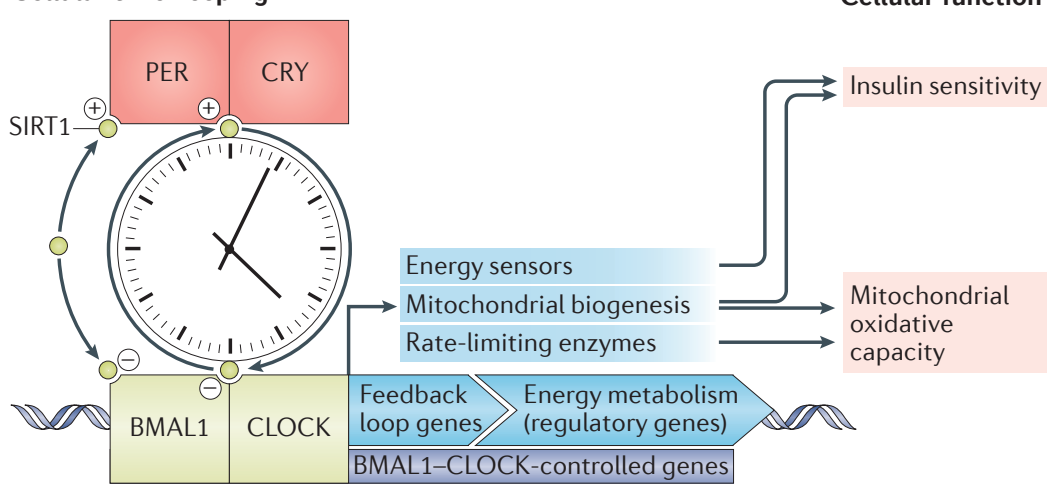

Figure 4 | Circadian clock and mitochondrial function. The cellular clockwork orchestrates circadian rhythms through oscillations in the transcription and translation of circadian locomoter output cycles protein kaput (CLOCK) and aryl hydrocarbon receptor nuclear translocator-like protein 1 (better known as BMAL1), which affect the levels of the BMAL1-CLOCK heterodimer. The self-regulation of the cycle occurs by BMAL1-CLOCK-controlled induction of period circadian protein homologue 1 (PER) and cryptochrome-2 (CRY) protein expression, after which the PER-CRY heterodimer triggers CLOCK and BMAL1 degradation. Furthermore, sirtuin 1 (SIRT1) activation can enhance the activity of PER-CRY and inhibits the activity of BMAL1. The activity of this peripheral clock leads to the transcription of genes involved in energy metabolism, which might affect insulin sensitivity and mitochondrial function.

general population, as artificial light enables individuals to avoid adapting their lifestyle to the circadian clock and plan activities independent of the day-night cycle.

Interestingly, research in the past decade clearly indicates that mitochondrial metabolism is under control of the biological clock and that peripheral tissues have their own rhythm, triggered by a cellular molecular clock. This molecular clock comprises closely intertwined transcriptional and post-translational negative feedback loops. Positive regulation of these feedback loops occurs via the transcription factors circadian locomoter output cycles protein kaput (CLOCK) and aryl hydrocarbon receptor nuclear translocator-like protein 1 (ARNTL, better known as BMAL1), which in turn activate the negative regulators cryptochrome-2 (CRY) and period circadian protein homologue 1 (PER) ${ }^{125}$. Downstream of BMAL1 is nuclear receptor subfamily 1 group D member 1 (NR1D1, also known as Rev-erbA- $\alpha$ ), an important nuclear receptor for lipid metabolism and adipogenesis $^{126}$, which is also expressed in skeletal muscle where it seems to modulate the oxidative phenotype ${ }^{127}$ (FIG. 4). Output of this molecular clock in peripheral tissues is very sensitive to feeding-fasting cycles and the nutrient sensors AMPK and SIRT1 are regulated in a circadian manner to modulate the rhythm of metabolism optimally in anticipation of periods of activity or food intake ${ }^{128}$. In mice and rats, circadian misalignment leads to mitochondrial dysfunction ${ }^{129}$ and genetically or pharmacologically stimulating increased NR1D1 levels and activity results in an 'athlete-like' phenotype, with induced mitochondrial biogenesis, improved mitochondrial respiratory capacity and increased running capacity ${ }^{127}$, factors commonly associated with improved insulin sensitivity. Intriguingly, upon liver-specific deletion of BMAL1 in a mouse model, liver mitochondria lose their capacity to adapt to altered nutrient availability, indicating a loss in metabolic flexibility ${ }^{130}$, a phenomenon that was rescued by genetically inducing mitochondrial fission ${ }^{130}$. Hence, it is likely that disturbances in the molecular clock in peripheral tissues underlie the strong, albeit indirect, epidemiological indications by which sleep deprivation and disruption of circadian rhythm in humans negatively affect mitochondrial function and insulin sensitivity. However, studies examining the direct involvement of the molecular clock and its downstream targets in regulating mitochondrial function in humans are warranted before the molecular clock can be identified as a therapeutic target to promote insulin sensitivity via mitochondrial function.

Carnitine supplementation. As outlined earlier in the article, T2DM, but also prediabetes, is characterized by metabolic inflexibility. In mouse and rat studies, insulin resistance and metabolic inflexibility have been associated with changes in carnitine status ${ }^{131}$. Processes such as ageing and the consumption of a high-fat diet, both recognized inducers of insulin resistance and metabolic inflexibility, were found to compromise the availability of free carnitine in mice ${ }^{131}$, whereas supplementation with carnitine in mice fed high-fat diets completely restored insulin sensitivity ${ }^{131}$. In addition, beneficial effects of carnitine supplementation on insulin sensitivity and glucose tolerance have been reported in humans ${ }^{132}$. As outlined previously in this article, in the presence of ample free carnitine, the mitochondrial enzyme CrAT can maintain the conversion of carnitine and acetyl-CoA to acetylcarnitine, reducing acetyl-CoA levels even under conditions of substrate oversupply. In the 1960s, it was proposed that a high level of acetyl-CoA inhibits pyruvate dehydrogenase activity, thereby reducing glucose catabolism, which could contribute to the development of insulin resistance ${ }^{133}$. However, high acetyl-CoA levels might also operate as an acetyl donor for mitochondrial protein acetylation, which is emerging as an important mechanism of metabolic regulation and mitochondrial function. In fact, protein hyperacetylation has been associated with insulin resistance upon high-fat feeding in mice ${ }^{134}$ and ageing ${ }^{135}$ in rats. Mitochondrial matrix proteins were shown to be sensitive to nutrient-induced protein acetylation, which is enhanced in mice lacking CrAT, suggesting that carnitine metabolism might also be involved in regulation of mitochondrial protein acetylation $^{136}$. Clearly, human intervention studies are needed to determine whether carnitine supplementation in patients can improve insulin sensitivity and mitochondrial function via attenuation of mitochondrial protein acetylation by stimulating acetylcarnitine synthesis.

Ambient temperature and cold exposure. The discovery of cold-inducible BAT in adult humans ${ }^{137}$ has resulted in a large number of patient and animal studies investigating whether activation of BAT could be considered as a therapeutic target to treat obesity and $\mathrm{T} 2 \mathrm{DM}^{138,139}$. Animal data reveal that cold exposure can reverse fastinginduced insulin resistance ${ }^{140}$ and that the beneficial effects of cold exposure on glucose homeostasis and insulin sensitivity are dependent on BAT mass ${ }^{141}$. 
However, the relative contribution of BAT to wholebody metabolism differs considerably between mice and humans. In fact, our group showed that 10 days of cold acclimation markedly promoted whole-body insulin sensitivity in patients who have T2DM, with an average increase in insulin sensitivity of $\sim 43 \%{ }^{142}$. Most remarkably, this increase in insulin sensitivity could not be explained by BAT activity, which only increased marginally. Rather, 10 days of cold acclimation resulted in a clear increase in GLUT-4 translocation to the cell surface in skeletal muscle, a finding that we also confirmed in patients with obesity ${ }^{143}$. So far, the underlying mechanism for this effect has not been unravelled, but did not involve substantial improvements in skeletal muscle mitochondrial respiration capacity.

In mice, cold-induced nonshivering thermogenesis in skeletal muscle requires activation of sarcolipin ${ }^{144}$ (a pivotal component of sarcoplasmic reticulum $\mathrm{Ca}^{2+}$-ATPase activity that is involved in mitochondrial biogenesis) and overexpression of this protein also increased fat oxidative capacity and energy expenditure in these animals ${ }^{145}$. Although under normal physiological conditions the energy needed for active $\mathrm{Ca}^{2+}$ transport is unlikely to exceed $10 \%$ of total muscle energy expenditure ${ }^{146}$, muscle energy expenditure might rise to $140 \%$ of normal conditions when the $\mathrm{Ca}^{2+}$ transient is slightly elevated (subcontraction depolarization of the sarcolemma) ${ }^{147}$. Notably, at this sub-contraction level of depolarization, muscle tension only increases by a mere $3 \%{ }^{147}$. Human studies examining the role of $\mathrm{Ca}^{2+}$ transients in energy expenditure, mitochondrial function and insulin sensitivity in skeletal muscle are presently lacking. However, induction of futile cycling via $\mathrm{Ca}^{2+}$ transients, be it by cold exposure or by sub-threshold muscle contractions, could contribute to increased energy expenditure and improved insulin sensitivity, as observed in humans upon cold exposure ${ }^{142}$, fidgeting ${ }^{148}$ or during very mild exercise regimes such as strolling ${ }^{149}$ or breaking sitting time ${ }^{150}$. Clearly, future studies are needed to expand this exciting field of research.

Ambient temperature and heat stress. Intriguingly, in addition to the association between cold exposure and improvements in insulin sensitivity, heat stress has also been linked to altered glucose homeostasis. Upon 3 weeks of heat exposure in a hot tub $\left(37-40^{\circ} \mathrm{C}\right)$ for $30 \mathrm{~min}$ per day, fasting plasma glucose concentration and $\mathrm{HbA}_{1 \mathrm{c}}$ levels dropped substantially in five male and three female patients with $\mathrm{T} 2 \mathrm{DM}^{151}$. A member of the family of heat responsive proteins known as heat shock proteins (HSPs), heat shock-related $70 \mathrm{kDa}$ protein 2 (HSPA2), seems to be involved in the glucoregulatory potential of heat exposure. HSPA2 levels were lower in skeletal muscle biopsy samples from patients with T2DM than in nondiabetic control individuals, with HSPA2 gene expression and protein content also found to correlate positively with insulin-stimulated glucose uptake ${ }^{152,153}$. More direct studies revealed that using heat stress, as well as pharmacological and genetic approaches, to stimulate HSPA2 expression all improved insulin sensitivity ${ }^{154}$. Moreover, heat-stress-mediated induction of HSPA2 in rats fed a high-fat diet alleviated insulin resistance, reduced mitochondrial dysfunction ${ }^{155}$ and improved both respiratory capacity and fat oxidative capacity ${ }^{155}$. Furthermore, induction of HSPA2 expression also induced mitochondrial biogenesis in an AMPK-SIRT1-PGC-1 1 dependent manner in cultured myotubes ${ }^{156}$.

A variety of small molecule activators and co-activators of HSPs seem to affect mitochondrial physiology ${ }^{157}$. Promisingly, one of these co-activators, a small molecule drug candidate named BGP-15, was proven to promote insulin sensitivity in nondiabetic individuals with insulin resistance ${ }^{158}$. Although this study did not examine direct involvement of HSPA2 or mitochondrial adaptations in the observed improvements in glucose handling, it seems fair to conclude that, in addition to cold exposure, mild heat stress might also affect mitochondrial function and improve insulin sensitivity.

\section{Conclusion and future perspectives}

The association between mitochondrial function and insulin sensitivity has been well established and ample evidence exists that boosting mitochondrial function improves whole-body and skeletal muscle insulin sensitivity and glucose homeostasis, at least in mice and rats. Albeit more limited in number than animal model studies, most human studies also provide compelling evidence that therapeutically targeting mitochondria to improve insulin sensitivity is a promising route. Importantly, mitochondria are central in the regulation of human cellular metabolism and cells relevant to metabolic control are equipped with robust regulatory pathways enabling them to adapt mitochondrial metabolism to cellular conditions and demands. These findings identify mitochondria as targets for pharmacological or exercise interventions to treat T2DM, and also suggest that these organelles can be manipulated by lifestyle factors such as nutrition, environmental temperature and circadian rhythms. Despite the abundance of animal data, mechanistic human clinical intervention studies to target the diverse aspects of mitochondrial function in the treatment or prevention of T2DM are still limited, but urgently awaited.
1. Navarro, A. \& Boveris, A. The mitochondrial energy transduction system and the aging process. $\mathrm{Am}$. J. Physiol. Cell Physiol. 292, C670-C686 (2007).

2. Shigenaga, M. K., Hagen, T. M. \& Ames, B. N.

Oxidative damage and mitochondrial decay in aging Proc. Natl Acad. Sci. USA 91, 10771-10778 (1994).

3. Cortopassi, G. A. \& Wong, A. Mitochondria in organismal aging and degeneration. Biochim. Biophys. Acta 1410, 183-193 (1999).
4. Carter, H., Chen, C. \& Hood, D. Mitochondria, muscle health, and exercise with advancing age. Physiology 30, 208-223 (2015)

5. Hood, D Uguccioni, G Vainshtein, A \& D'souza, D. Mechanisms of exercise-induced mitochondrial biogenesis in skeletal muscle: implications for health and disease. Compr. Physiol. 1, 1119-1134 (2011).

6. Shulman, G. I. et al. Quantitation of muscle glycogen synthesis in normal subjects and subjects with non- insulin-dependent diabetes by ${ }^{13} \mathrm{C}$ nuclear magnetic resonance spectroscopy. N. Engl. J. Med. 322, 223-228 (1990)

7. Unger, R. H. \& Orci, L. Diseases of liporegulation: new perspective on obesity and related disorders. FASEB J. 15, 312-321 (2001)

8. Kelley, D. E., He, J., Menshikova, E. V. \& Ritov, V. B. Dysfunction of mitochondria in human skeletal muscle in type 2 diabetes. Diabetes 51, 2944-2950 (2002). 
9. Ritov, V. B. et al. Deficiency of subsarcolemmal mitochondria in obesity and type 2 diabetes. Diabetes 54, 8-14 (2005)

10. Mootha, V. K. et al. PGC-1 $\alpha$-responsive genes involved in oxidative phosphorylation are coordinately downregulated in human diabetes. Nat. Genet. 34 267-273 (2003).

11. Patti, M. E. et al. Coordinated reduction of genes of oxidative metabolism in humans with insulin resistance and diabetes: potential role of $P G C 1$ and NRF1. Proc. Natl Acad. Sci. USA 100, 8466-8471 (2003).

12. Petersen, K. F. et al. Mitochondrial dysfunction in the elderly: possible role in insulin resistance. Science 300, 1140-1142 (2003)

13. Petersen, K. F., Dufour, S., Befroy, D., Garcia, R. \& Shulman, G. I. Impaired mitochondrial activity in the insulin-resistant offspring of patients with type 2 diabetes. N. Engl. J. Med. 350, 664-671 (2004).

14. Kemp, G. \& Brindle, K. What do magnetic resonancebased measurements of $\mathrm{Pi} \rightarrow$ ATP flux tell us about skeletal muscle metabolism? Diabetes 61 , 1927-1934 (2012).

15. Schrauwen-Hinderling, V. B. et al. Impaired in vivo mitochondrial function but similar intramyocellular lipid content in patients with type 2 diabetes mellitus and BMI-matched control subjects. Diabetologia 50 , 113-120 (2007)

16. Phielix, E. et al. Lower intrinsic ADP-stimulated mitochondrial respiration underlies in vivo mitochondrial dysfunction in muscle of male type 2 diabetic patients. Diabetes 57, 2943-2949 (2008).

17. Hancock, C. R. et al. High-fat diets cause insulin resistance despite an increase in muscle mitochondria. Proc. Natl Acad. Sci. USA 105 7815-7820 (2008)

18. Turner, N. et al. Excess lipid availability increases mitochondrial fatty acid oxidative capacity in muscle: evidence against a role for reduced fatty acid oxidation in lipid-induced insulin resistance in rodents. Diabetes 56, 2085-2092 (2007).

19. Pospisilik, J. A. et al. Targeted deletion of AIF decreases mitochondrial oxidative phosphorylation and protects from obesity and diabetes. Cell 131 , 476-491 (2007)

20. Schiff, M. et al. Mitochondria and diabetes mellitus: untangling a conflictive relationship? J. Inherit. Metab. Dis. 32, 684-698 (2009)

21. Dumas, J., Simard, G., Flamment, M., Ducluzeau, P. \& Ritz, P. Is skeletal muscle mitochondrial dysfunction a cause or an indirect consequence of insulin resistance in humans? Diabetes Metab. 35 159-167 (2009).

22. Joseph, A. \& Hood, D. Relationships between exercise, mitochondrial biogenesis and type 2 diabetes. Med. Sport Sci. 60, 48-61 (2014).

23. Toledo, F. \& Goodpaster, B. The role of weight loss and exercise in correcting skeletal muscle mitochondria abnormalities in obesity, diabetes and aging $\mathrm{Mol}$. Cell. Endocrinol. 379, 30-34 (2013).

24. Brunmair, B. et al. Thiazolidinediones, like metformin, inhibit respiratory complex I: a common mechanism contributing to their antidiabetic actions? Diabetes 53, 1052-1059 (2004)

25. Madiraju, A. K. et al. Metformin suppresses gluconeogenesis by inhibiting mitochondrial glycerophosphate dehydrogenase. Nature $\mathbf{5 1 0}$ 542-546 (2014)

26. Matsuzaki, S. \& Humphries, K. M. Selective inhibition of deactivated mitochondrial complex I by biguanides. Biochemistry 54, 2011-2021 (2015)

27. Satapati, S. et al. Mitochondrial metabolism mediates oxidative stress and inflammation in fatty liver. J. Clin Invest. 125, 4447-4462 (2015).

28. Romanello, V. \& Sandri, M. Mitochondrial quality control and muscle mass maintenance. Front. Physiol. 6, 422 (2016)

29. Thomas, R. \& Gustafsson, A. Mitochondrial autophagy. Circ. J. 77, 2449-2454 (2013).

30. Jung, H. S. \& Lee, M. S. Role of autophagy in diabetes and mitochondria. Ann. NY Acad. Sci. 1201, 79-83 (2010).

31. Randle, P., Garland, P., Hales, C. \& Newsholme, E. The glucose fatty-acid cycle its role in insulin sensitivity and the metabolic disturbances of diabetes mellitus. Lancet 281, 785-789 (1963).

32. Storlien, L., Oakes, N. \& Kelley, D. Metabolic flexibility. Proc. Nutr. Soc. 63, 363-368 (2004).

33. Kelley, D. E. \& Mandarino, L. J. Fuel selection in human skeletal muscle in insulin resistance: a reexamination. Diabetes 49, 677-683 (2000).
34. Mihalik, S. J. et al. Increased levels of plasma acylcarnitines in obesity and type 2 diabetes and identification of a marker of glucolipotoxicity. Obesity (Silver Spring) 18, 1695-1700 (2010).

35. Seiler, S. E. et al. Obesity and lipid stress inhibit carnitine acetyltransferase activity. J. Lipid Res. 55 , 635-644 (2014).

36. Lindeboom, L. et al. Long-echo time MR spectroscopy for skeletal muscle acetylcarnitine detection. J. Clin. Invest. 124, 4915-4925 (2014).

37. Song, F. et al. Oxidative stress, antioxidant status and DNA damage in patients with impaired glucose regulation and newly diagnosed type 2 diabetes. Clin. Sci. (Lond.) 112, 599-606 (2007).

38. Anderson, E. J. et al. Mitochondrial $\mathrm{H}_{2} \mathrm{O}_{2}$ emission and cellular redox state link excess fat intake to insulin resistance in both rodents and humans. J. Clin. Invest. 119, 573-581 (2009)

39. Amara, C. E. et al. Mild mitochondrial uncoupling impacts cellular aging in human muscles in vivo. Proc. Natl Acad. Sci. USA 104, 1057-1062 (2007).

40. Diehl, A. M. \& Hoek, J. B. Mitochondrial uncoupling: role of uncoupling protein anion carriers and relationship to thermogenesis and weight control "the benefits of losing control". J. Bioenerg. Biomembr. 31, 493-506 (1999).

41. Wallin, I. E. The mitochondria problem. Am. Naturalist 57, 255-261 (1923).

42. Schatz, G., Haslbrunner, E. \& Tuppy, H. Deoxyribonucleic acid associated with yeast mitochondria. Biochem. Biophys. Res. Commun. 15 127-132 (1964)

43. Lee, C., Yen, K. \& Cohen, P. Humanin: a harbinger of mitochondrial-derived peptides? Trends Endocrinol. Metab. 24, 222-228 (2013).

44. Lee, C. et al. The mitochondrial-derived peptide MOTS-c promotes metabolic homeostasis and reduces obesity and insulin resistance. Cell Metab. 21 443-454 (2015).

45. Mottis, A., Jovaisaite, V. \& Auwerx, J. The mitochondrial unfolded protein response in mammalian physiology. Mamm. Genome $\mathbf{2 5}$, 424-433 (2014).

46. Ljubicic, V. et al. Transcriptional and posttranscriptional regulation of mitochondrial biogenesis in skeletal muscle: effects of exercise and aging. Biochim. Biophys. Acta 1800, 223-234 (2010)

47. Lin, J. et al. Transcriptional co-activator PGC- $1 \alpha$ drives the formation of slow-twitch muscle fibres. Nature 418, 797-801 (2002)

48. Hoeks, J. et al. Enhanced lipid-but not carbohydratesupported mitochondrial respiration in skeletal muscle of PGC- $1 \alpha$ overexpressing mice. J. Cell. Physiol. 227, 1026-1033 (2012)

49. Choi, C. S et al. Paradoxical effects of increased expression of PGC- $1 \alpha$ on muscle mitochondrial function and insulin-stimulated muscle glucose metabolism. Proc. Natl Acad. Sci. USA 105 19926-19931 (2008)

50. Summermatter, S. et al. PGC-1 $\alpha$ improves glucose homeostasis in skeletal muscle in an activitydependent manner. Diabetes 62, 85-95 (2013)

51. Koves, T. R. et al. PPAR $\gamma$ coactivator- $1 \alpha$ contributes to exercise-induced regulation of intramuscular lipid droplet programming in mice and humans. J. Lipid Res. 54, 522-534 (2013).

52. Meex, R. C. et al. Restoration of muscle mitochondrial function and metabolic flexibility in type 2 diabetes by exercise training is paralleled by increased myocellular fat storage and improved insulin sensitivity. Diabetes 59, 572-579 (2010).

53. Mensink, M. et al. Improved skeletal muscle oxidative enzyme activity and restoration of PGC- $1 \alpha$ and $\mathrm{PPAR} \beta / \delta$ gene expression upon rosiglitazone treatment in obese patients with type 2 diabetes mellitus. Int. J. Obes. (Lond.) 31, 1302-1310 (2007).

54. Canto, C. \& Auwerx, J. PGC-1 $\alpha$, SIRT 1 and AMPK, an energy sensing network that controls energy expenditure. Curr. Opin. Lipidol. 20, 98-105 (2009).

55. Zorzano, A., Hernandez-Alvarez, M. I., Palacin, M. \& Mingrone, G. Alterations in the mitochondrial regulatory pathways constituted by the nuclear co-factors PGC- $1 \alpha$ or PGC- $1 \beta$ and mitofusin 2 in skeletal muscle in type 2 diabetes. Biochim. Biophys. Acta 1797, 1028-1033 (2010).

56. Glancy, B. et al. Mitochondrial reticulum for cellular energy distribution in muscle. Nature 523, 617-620 (2015).

57. Westermann, B. Mitochondrial fusion and fission in cell life and death. Nat. Rev. Mol. Cell Biol. 11 872-884 (2010).
58. Cipolat, S., Martins de Brito, O., Dal Zilio, B. $\delta$ Scorrano, L. OPA1 requires mitofusin 1 to promote mitochondrial fusion. Proc. Natl Acad. Sci. USA 101, 15927-15932 (2004).

59. Herlan, M., Bornhovd, C., Hell, K., Neupert, W. \& Reichert, A. S. Alternative topogenesis of Mgm 1 and mitochondrial morphology depend on ATP and a functional import motor. J. Cell Biol. 165, 167-173 (2004).

60. Tondera, D et al SLP-2 is required for stress-induced mitochondrial hyperfusion. EMBO J. 28, 1589-1600 (2009).

61. Patten, D. A. et al. OPA 1-dependent cristae modulation is essential for cellular adaptation to metabolic demand. EMBO J. 33, 2676-2691 (2014).

62. Liesa, M. \& Shirihai, O. S. Mitochondrial dynamics in the regulation of nutrient utilization and energy expenditure. Cell Metab. 17, 491-506 (2013).

63. Lee, Y. J., Jeong, S. Y., Karbowski, M., Smith, C. L. $\&$ Youle, R. J. Roles of the mammalian mitochondrial fission and fusion mediators Fis 1 , Drp 1, and Opa 1 in apoptosis. Mol. Biol. Cell 15, 5001-5011 (2004).

64. Wikstrom, J. D. et al. Hormone-induced mitochondrial fission is utilized by brown adipocytes as an amplification pathway for energy expenditure. EMBO J. 33, 418-436 (2014).

65. Poehlman, E. T. \& Horton, E. S. Regulation of energy expenditure in aging humans. Annu. Rev. Nutr. 10, 255-275 (1990)

66. Schrauwen, P., Xia, J., Bogardus, C., Pratley, R. E. \& Ravussin, E. Skeletal muscle uncoupling protein 3 expression is a determinant of energy expenditure in Pima Indians. Diabetes 48, 146-149 (1999).

67. Twig, G. et al. Fission and selective fusion govern mitochondrial segregation and elimination by autophagy. EMBO J. 27, 433-446 (2008).

68. Sebastian, D. et al. Mitofusin 2 (Mfn2) links mitochondrial and endoplasmic reticulum function with insulin signaling and is essential for normal glucose homeostasis. Proc. Natl Acad. Sci. USA 109 5523-5528 (2012).

69. Pich, S. et al. The Charcot-Marie-Tooth type 2A gene product, Mfn2, up-regulates fuel oxidation through expression of OXPHOS system. Hum. Mol. Genet. 14 1405-1415 (2005).

70. Zuchner, S. et al. Mutations in the mitochondrial GTPase mitofusin 2 cause Charcot-Marie-Tooth neuropathy type 2A. Nat. Genet. 36, 449-451 (2004).

71. Bach, D. et al. Expression of Mfn2, the CharcotMarie-Tooth neuropathy type $2 \mathrm{~A}$ gene, in human skeletal muscle. Diabetes 54, 2685-2693 (2005).

72. Jheng, H. F. et al. Mitochondrial fission contributes to mitochondrial dysfunction and insulin resistance in skeletal muscle. Mol. Cell. Biol. 32, 309-319 (2012).

73. Watanabe, T. et al. Roles of mitochondrial fragmentation and reactive oxygen species in mitochondrial dysfunction and myocardial insulin resistance. Exp. Cell Res. 323, 314-325 (2014).

74. Waterham, H. R. et al. A lethal defect of mitochondrial and peroxisomal fission. N. Engl. J. Med. 356, 1736-1741 (2007)

75. Wallberg-Henriksson, H. et al. Increased peripheral insulin sensitivity and muscle mitochondrial enzymes but unchanged blood glucose control in type I diabetics after physical training. Diabetes 31 1044-1050 (1982).

76. James, D. E., Lederman, L. \& Pilch, P. F. Purification of insulin-dependent exocytic vesicles containing the glucose transporter. J. Biol. Chem. 262, 11817-11824 (1987).

77. Menshikova, E. V. et al. Effects of weight loss and physical activity on skeletal muscle mitochondrial function in obesity. Am. J. Physiol. Endocrinol. Metab. 288, E818-E825 (2005).

78. Short, K. R. et al. Impact of aerobic exercise training on age-related changes in insulin sensitivity and muscle oxidative capacity. Diabetes 52, 1888-1896 (2003).

79. Toledo, F. G. et al. Effects of physical activity and weight loss on skeletal muscle mitochondria and relationship with glucose control in type 2 diabetes. Diabetes 56, 2142-2147 (2007)

80. Bajpeyi, S. et al. Skeletal muscle mitochondrial capacity and insulin resistance in type 2 diabetes. J. Clin. Endocrinol. Metab. 96, 1160-1168 (2011). 
81. Hutchison, S. K. et al. Effect of exercise training on insulin sensitivity, mitochondria and computed tomography muscle attenuation in overweight women with and without polycystic ovary syndrome. Diabetologia 55, 1424-1434 (2012).

82. Hey-Mogensen, M. et al. Effect of physical training on mitochondrial respiration and reactive oxygen species release in skeletal muscle in patients with obesity and type 2 diabetes. Diabetologia 53, 1976-1985 (2010).

83. Hood, M. S., Little, J. P., Tarnopolsky, M. A., Myslik, F. ¿ Gibala, M. J. Low-volume interval training improves muscle oxidative capacity in sedentary adults. Med. Sci. Sports Exerc. 43, 1849-1856 (2011).

84. Irving, B. A., Short, K. R., Nair, K. S. \& Stump, C. S. Nine days of intensive exercise training improves mitochondrial function but not insulin action in adult offspring of mothers with type 2 diabetes. J. Clin. Endocrinol. Metab. 96, E1137-E1141 (2011).

85. Coen, P. M. et al. Exercise and weight loss improve muscle mitochondrial respiration, lipid partitioning, and insulin sensitivity after gastric bypass surgery. Diabetes 64, 3737-3750 (2015).

86. Phielix, E., Meex, R., Moonen-Kornips, E. Hesselink, M. K. \& Schrauwen, P. Exercise training increases mitochondrial content and ex vivo mitochondrial function similarly in patients with type 2 diabetes and in control individuals. Diabetologia 53 , 1714-1721 (2010)

87. Pagel-Langenickel, I. et al. A discordance in rosiglitazone mediated insulin sensitization and skeletal muscle mitochondrial content/activity in Type 2 diabetes mellitus. Am. J. Physiol. Heart Circ. Physiol. 293, H2659-H2666 (2007).

88. Malin, S. K. et al. Insulin sensitivity and metabolic flexibility following exercise training among different obese insulin-resistant phenotypes. Am. J. Physiol. Endocrinol. Metab. 305, E1292-E1 298 (2013).

89. Bergouignan, A. et al. Effect of contrasted levels of habitual physical activity on metabolic flexibility. J. Appl. Physiol. (1985) 114, 371-379 (2013).

90. den Hoed, M., Hesselink, M. K., van Kranenburg, G. P. \& Westerterp, K. R. Habitual physical activity in daily life correlates positively with markers for mitochondrial capacity. J. Appl. Physiol. (1985) 105 561-568 (2008)

91. Karolkiewicz, J. et al. Response of oxidative stress markers and antioxidant parameters to an 8-week aerobic physical activity program in healthy, postmenopausal women. Arch. Gerontol. Geriatr. 49 e67-e71 (2009)

92. Konopka, A. R. et al. Defects in mitochondrial efficiency and $\mathrm{H}_{2} \mathrm{O}_{2}$ emissions in obese women are restored to a lean phenotype with aerobic exercise training. Diabetes 64, 2104-2115 (2015)

93. Ludzki, A. et al. Rapid repression of ADP transport by palmitoyl-CoA is attenuated by exercise training in humans: a potential mechanism to decrease oxidative stress and improve skeletal muscle insulin signaling. Diabetes 64, 2769-2779 (2015).

94. Mielke, C. et al. Adenine nucleotide translocase is acetylated in vivo in human muscle: modeling predicts a decreased ADP affinity and altered control of oxidative phosphorylation. Biochemistry 53 3817-3829 (2014).

95. Sparks, L. M. et al. Ant1-mediated fatty acid-induced uncoupling as a target for improving myocellular insulin sensitivity. Diabetologia 59, 1030-1039 (2016).

96. Befroy, D. E. et al. Increased substrate oxidation and mitochondrial uncoupling in skeletal muscle of endurance-trained individuals. Proc. Natl Acad. Sci. USA 105, 16701-16706 (2008)

97. Ding, H. et al. Response of mitochondrial fusion and fission protein gene expression to exercise in rat skeletal muscle. Biochim. Biophys. Acta 1800 250-256 (2010)

98. Cartoni, R. et al. Mitofusins $1 / 2$ and ERR $\alpha$ expression are increased in human skeletal muscle after physical exercise. J. Physiol. 567, 349-358 (2005).

99. Garnier, A. et al. Coordinated changes in mitochondrial function and biogenesis in healthy and diseased human skeletal muscle. FASEB J. 19, 43-52 (2005).

100. Hernandez-Alvarez, M. I. et al. Subjects with earlyonset type 2 diabetes show defective activation of the skeletal muscle PGC- $1 \alpha /$ mitofusin- 2 regulatory pathway in response to physical activity. Diabetes Care 33, 645-651 (2010)
101. Fealy, C. E., Mulya, A., Lai, N. \& Kirwan, J. P. Exercise training decreases activation of the mitochondrial fission protein dynamin-related protein-1 in insulinresistant human skeletal muscle. J. Appl. Physiol. (1985) 117, 239-245 (2014)

102. Finkel, T. The metabolic regulation of aging. Nat. Med. 21, 1416-1423 (2015).

103. Heilbronn, L. K. et al. Effect of 6-month calorie restriction on biomarkers of longevity, metabolic adaptation, and oxidative stress in overweight individuals: a randomized controlled trial. JAMA 295. 1539-1548 (2006).

104. Civitarese, A. E. et al. Calorie restriction increases muscle mitochondrial biogenesis in healthy humans. PLoS Med. 4, e76 (2007)

105. Guarente, L. Mitochondria - a nexus for aging, calorie restriction, and sirtuins? Cell 132, 171-176 (2008)

106. Sun, Q. et al. Overexpression of visfatin/PBEF/Nampt alters whole-body insulin sensitivity and lipid profile in rats. Ann Med 41, 311-320 (2009).

107. Yoshino, J., Mills, K. F., Yoon, M. J. \& Imai, S. Nicotinamide mononucleotide, a key NAD ${ }^{+}$ intermediate, treats the pathophysiology of diet- and age-induced diabetes in mice. Cell Metab. 14 528-536 (2011)

108. Baur, J. A. et al. Resveratrol improves health and survival of mice on a high-calorie diet. Nature $\mathbf{4 4 4}$ 337-342 (2006)

109. Milne, J. C. et al. Small molecule activators of SIRT1 as therapeutics for the treatment of type 2 diabetes. Nature 450, 712-716 (2007).

110. Rutanen, J. et al. SIRT1 mRNA expression may be associated with energy expenditure and insulin sensitivity. Diabetes 59, 829-835 (2010).

111. Baksi, A. et al. A phase II, randomized, placebo controlled, double-blind, multi-dose study of SRT2104, a SIRT1 activator, in subjects with type 2 diabetes. Br. J. Clin. Pharmacol. 78, 69-77 (2014)

112. Howitz, K. T. et al. Small molecule activators of sirtuins extend Saccharomyces cerevisiae lifespan. Nature 425, 191-196 (2003)

113. de Ligt, M., Timmers, S. \& Schrauwen, P. Resveratro and obesity: can resveratrol relieve metabolic disturbances? Biochim. Biophys. Acta 1852 1137-1144 (2015)

114. Timmers, S. et al. Calorie restriction-like effects of 30 days of resveratrol supplementation on energy metabolism and metabolic profile in obese humans. Cell Metab. 14, 612-622 (2011).

115. Timmers, S., Hesselink, M. K. \& Schrauwen, P. Therapeutic potential of resveratrol in obesity and type 2 diabetes: new avenues for health benefits? Ann. NY Acad. Sci. 1290, 83-89 (2013)

116. Canto, C. et al. The $\mathrm{NAD}^{+}$precursor nicotinamide riboside enhances oxidative metabolism and protects against high-fat diet-induced obesity. Cell Metab. 15 838-847 (2012).

117. Gariani, K et al. Eliciting the mitochondrial unfolded protein response via NAD repletion reverses fatty liver disease. Hepatology 63 , 1190-1204 (2016)

118. Traba, J. et al. Fasting and refeeding differentially regulate NLRP3 inflammasome activation in human subjects. J. Clin. Invest. 125, 4592-4600 (2015)

119. van de Weijer, T. et al. Evidence for a direct effect of the $\mathrm{NAD}^{+}$precursor acipimox on muscle mitochondrial function in humans. Diabetes 64 1193-1201 (2015)

120. Pan, A., Schernhammer, E. S., Sun, Q. \& Hu, F. B. Rotating night shift work and risk of type 2 diabetes: two prospective cohort studies in women. PLoS Med. 8, e1001141 (2011)

121. Suwazono, Y. et al. Shiftwork and impaired glucose metabolism: a 14-year cohort study on 7104 male workers. Chronobiol. Int. 26, 926-941 (2009).

122. Meisinger, C., Heier, M. \& Loewel, H. Sleep disturbance as a predictor of type 2 diabetes mellitus in men and women from the general population. Diabetologia 48, 235-241 (2005).

123. Scheer, F. A., Hilton, M. F., Mantzoros, C. S. \& Shea, S. A. Adverse metabolic and cardiovascular consequences of circadian misalignment. Proc. Natl Acad. Sci. USA 106, 4453-4458 (2009).

124. Karlsson, B., Knutsson, A. \& Lindahl, B. Is there an association between shift work and having a metabolic syndrome? Results from a population based study of 27,485 people. Occup. Environ. Med. 58, 747-752 (2001).
125. Kalsbeek, A., la Fleur, S. \& Fliers, E. Circadian control of glucose metabolism. Mol. Metab. 3, 372-383 (2014).

126. Yin, L., Wang, J., Klein, P. S. \& Lazar, M. A. Nuclear receptor Rev-erb $\alpha$ is a critical lithium-sensitive component of the circadian clock. Science 311 1002-1005 (2006)

127. Woldt, E. et al. Rev-erb- $\alpha$ modulates skeletal muscle oxidative capacity by regulating mitochondrial biogenesis and autophagy. Nat. Med. 19, 1039-1046 (2013)

128. Eckel-Mahan, K. L. et al. Reprogramming of the circadian clock by nutritional challenge. Cell 155 1464-1478 (2013).

129. Peek, C. B. et al. Circadian clock $\mathrm{NAD}^{+}$cycle drives mitochondrial oxidative metabolism in mice. Science 342, 1243417 (2013).

130. Jacobi, D. et al. Hepatic Bmal 1 regulates rhythmic mitochondrial dynamics and promotes metabolic fitness. Cell Metab. 22, 709-720 (2015).

131. Noland, R. C. et al. Carnitine insufficiency caused by aging and overnutrition compromises mitochondrial performance and metabolic control. J. Biol. Chem. 284, 22840-22852 (2009).

132. Ringseis, R., Keller, J. \& Eder, K. Role of carnitine in the regulation of glucose homeostasis and insulin sensitivity: evidence from in vivo and in vitro studies with carnitine supplementation and carnitine deficiency. Eur. J. Nutr. 51, 1-18 (2012).

133. Randle, P. J., Garland, P. B., Hales, C. N. \& Newsholme, E. A. The glucose fatty-acid cycle. Its role in insulin sensitivity and the metabolic disturbances of diabetes mellitus. Lancet 1, 785-789 (1963).

134. Hirschey, M. D. et al. SIRT3 deficiency and mitochondrial protein hyperacetylation accelerate the development of the metabolic syndrome. Mol. Cell 44 177-190 (2011)

135. Marton, O. et al. Aging and exercise affect the level of protein acetylation and SIRT 1 activity in cerebellum of male rats. Biogerontology 11 679-686 (2010)

136. Davies, M. J. et al. The acetyl group buffering action of carnitine acetyltransferase offsets macronutrientinduced lysine acetylation of mitochondrial proteins Cell Rep. 14, 1-12 (2016).

137. van Marken Lichtenbelt, W. D. et al. Cold-activated brown adipose tissue in healthy men. N. Engl. J. Med. 360, 1500-1508 (2009)

138. Betz, M. J. \& Enerback, S. Human brown adipose tissue: what we have learned so far. Diabetes 64 2352-2360 (2015)

139. Kajimura, S., Spiegelman, B. M. \& Seale, P. Brown and beige fat: physiological roles beyond heat generation. Cell Metab. 22, 546-559 (2015).

140. Shibata, H., Perusse, F., Vallerand, A. \& Bukowiecki, L. J. Cold exposure reverses inhibitory effects of fasting on peripheral glucose uptake in rats. Am. J. Physiol. 257, R96-R101 (1989).

141. Stanford, K. I. et al. Brown adipose tissue regulates glucose homeostasis and insulin sensitivity. J. Clin. Invest. 123, 215-223 (2013).

142. Hanssen, M. J. et al. Short-term cold acclimation improves insulin sensitivity in patients with type 2 diabetes mellitus. Nat. Med. 21, 863-865 (2015).

143. Hanssen, M. J. et al. Short-term cold acclimation recruits brown adipose tissue in obese humans. Diabetes 65, 1179-1189 (2016)

144. Bal, N. C. et al. Sarcolipin is a newly identified regulator of muscle-based thermogenesis in mammals. Nat. Med. 18, 1575-1579 (2012).

145. Maurya, S. K. et al. Sarcolipin is a key determinant of the basal metabolic rate, and its overexpression enhances energy expenditure and resistance against diet-induced obesity. J. Biol. Chem. 290 10840-10849 (2015).

146. Milligan, L. P. \& McBride, B. W. Energy costs of ion pumping by animal tissues. J. Nutr. 115, 1374-1382 (1985).

147. Chinet, A., Decrouy, A. \& Even, P. C. Ca(2+) dependent heat production under basal and nearbasal conditions in the mouse soleus muscle. J. Physiol. 455, 663-678 (1992).

148. Levine, J. A. et al. Interindividual variation in posture allocation: possible role in human obesity. Science 307, 584-586 (2005).

149. van Dijk, J. W. et al. Effect of moderate-intensity exercise versus activities of daily living on 24-hou blood glucose homeostasis in male patients with type 2 diabetes. Diabetes Care 36, 3448-3453 (2013). 
150. Henson, J. et al. Breaking up prolonged sitting with standing or walking attenuates the postprandial metabolic response in postmenopausal women: a randomized acute study. Diabetes Care 39, 130-138 (2016).

151. Hooper, P. L. Hot-tub therapy for type 2 diabetes mellitus. N. Engl. J. Med. 341, 924-925 (1999).

152. Bruce, C. R., Carey, A. L., Hawley, J. A. \& Febbraio, M. A. Intramuscular heat shock protein 72 and heme oxygenase- 1 mRNA are reduced in patients with type 2 diabetes: evidence that insulin resistance is associated with a disturbed antioxidant defense mechanism. Diabetes 52, 2338-2345 (2003).

153. Kurucz, I. et al. Decreased expression of heat shock protein 72 in skeletal muscle of patients with type 2 diabetes correlates with insulin resistance. Diabetes 51, 1102-1109 (2002)

154. Chung, J. et al. HSP72 protects against obesityinduced insulin resistance. Proc. Natl Acad. Sci. USA 105, 1739-1744 (2008).
155. Gupte, A. A., Bomhoff, G. L., Swerdlow, R. H. \& Geiger, P. C. Heat treatment improves glucose tolerance and prevents skeletal muscle insulin resistance in rats fed a high-fat diet. Diabetes $\mathbf{5 8}$, 567-578 (2009).

156. Liu, C. T. \& Brooks, G. A. Mild heat stress induces mitochondrial biogenesis in C2C12 myotubes. J. Appl. Physiol. (1985) 112, 354-361 (2012).

157. Henstridge, D. C., Whitham, M. \& Febbraio, M. A Chaperoning to the metabolic party: the emerging therapeutic role of heat-shock proteins in obesity and type 2 diabetes. Mol. Metab. 3, 781-793 (2014).

158. Literati-Nagy, B. et al. Improvement of insulin sensitivity by a novel drug, BGP-15, in insulinresistant patients: a proof of concept randomized double-blind clinical trial. Horm. Metab. Res. 41, 374-380 (2009)

159. Nabben, M. et al. The effect of UCP3 overexpression on mitochondrial ROS production in skeletal muscle of young versus aged mice. FEBS Lett. 582, 4147-4152 (2008).
160. Schrauwen, P. \& Hesselink, M. K. Oxidative capacity, lipotoxicity, and mitochondrial damage in type 2 diabetes. Diabetes 53, 1412-1417 (2004).

Acknowledgements

M.K.C.H. is supported by a VIDI Research Grant for innovative research from the Netherlands Organization for Scientific Research (Grant 917.66.359). V.S.-H. is supported by a VEN Research Grant for innovative research from the Netherlands Organization for Scientific Research (Grant 916.11.136). P.S is supported by a $\mathrm{VICl}$ Research Grant for innovative research from the Netherlands Organization for Scientific Research (Grant 918.96.618)

Author contributions

M.K.C.H. researched data and wrote the manuscript. All authors contributed to discussion of the content and reviewed and/or edited the article before submission.

Competing interests statement

The authors declare no competing interests. 